\title{
Potential Unconventional Medicines for the Treatment of SARS-CoV-2
}

Authors

Maurizio Coppola ${ }^{1}$, Raffaella Mondola ${ }^{2}$

Affiliations

1 Department of Addiction, ASL CN2, Alba, Italy

2 Department of Mental Health, ASL CN1, Saluzzo, Italy

Key words

antiviral drugs, pharmacology, drug research

To the Editor

SARS-CoV-2 infection was first isolated in Wuhan in the end of December 2019, since then, this $\beta$-coronavirus has rapidly spread becoming the most important public health concern worldwide. Numerous research Centers are working hard to realize a sure and effective medicine/vaccine as soon as possible, however the timing for having the availability of a curative/preventive treatments are unpredictable. Considering the severe health and economic consequences related with the SARS-CoV-2 pandemic, physicians are testing different antiretroviral and non-antiretroviral drugs for treating infected patients. Scientific literature review has shown interesting information about the potential antiretroviral action of some compounds not conventionally used for the treatment of virosis. Adem and colleagues in their virtual screening based molecular docking study reported a potential binding affinity exerted by various bioflavonoids at the active site of the MPro, the main protease of the SARS-CoV-2 [1]. Potential inhibition properties were studied using the Molegro Virtual Docker Program and some flavonoids, in particular hesperidin, rutin, and diosmin showed a better affinity for the MPro than nelfinavir [1]. Hesperidin showed the highest binding energy at the active site of the MPro with a MoLDock score of -178.5910 (nelfinavir-147.3800) and a HBond of-20.2594 (nelfinavir -6.8731). Moreover, the hesperidin could bind both Spike protein and ACE2 receptor preventing the development of the spikeACE2 complex used by the virus to enter cells [2]. Rutin showed the second strongest binding energy at the active site of the MPro with a MoLDock score of -176.2740 and a HBond of
-21.2402. Additionally, in preclinical studies the rutin reduced the level of interleukin 6 , a pro-inflammatory cytokine involved in the "cytokine storm" responsible for some severe infection-related complications [3]. Diosmin highlighted the third strongest binding energy at the active site of the MPro with a MoLDock score of -174.1260 and a HBond of -27.2572. As signaled for the rutin, in preclinical models the diosmin reduced the level of interleukin 6 as well as the number of CD4 and CD8 receptors in T cells [4]. Recently, Caly and colleagues reported a potential antiretroviral activity of the anti-parasitic agent ivermectin against SARS-CoV-2 virus. They demonstrated a reduction of about 5000 -fold of viral RNA in ivermectin-treated cells compared to control samples after 48 hours treatment. Ivermectin have showd antiviral effects against HIV, West Nile, Venezuelan equine encephalitis, and pseudorabies in previous preclinical and clinical studies [5]. Hoffmann and colleagues have further shown that SARS-CoV-2 uses the SARS-CoV receptor ACE2 for entry and the serine protease TMPRSS2 for Spike protein priming [6]. So, inhibition of the androgen-regulated serine protease TMPRSS2 activity could be a probable target for antiretroviral treatments. About that, bromhexine, a well tolerated mucolytic acting as cough suppressant, displayed to be able to inhibit the TMPRSS2 in a mice model study [7]. SARS-CoV-2 infection is causing thousands of victims worldwide by subjecting healthcare workers to an unprecedented effort. Despite the absence of clinical evidences regarding some potential anti-SARS-CoV-2 drugs, preliminar information reported by researchers suggest anyhow further studies in order to evaluate the clinical effects as well as the possibility to synthesize derivatives with stronger antiretroviral properties. Compounds reported in our paper have shown a safety profile for human and a potential anti-SARS-CoV2 activity.

\section{Conflict of Interest}

The authors declare that they have no conflict of interest.

\section{References}

[1] Adem S, Eyupoglu V, Sarfraz I et al. Identification of potent COVID-19 main protease (Mpro) inhibitors from natural polyphenols: An in silico strategy unveils a hope against corona. Preprints 2020; doi: 10.20944/preprints202003.0333.v1

[2] Wu C, Liu Y, Yang Y et al. Analysis of therapeutic targets for SARS-CoV-2 and discovery of potential drugs by computational methods. Acta Pharmaceutica Sinica B 2020; https://doi.org/10.1016/j.apsb.2020.02.008

[3] Liu S, Adewole D, Yu L et al. Rutin attenuates inflammatory responses induced by lipopolysaccharide in an in vitro mouse muscle cell (C2C12) model. Poult Sci 2019; 98: 2756-2764

[4] Imam F, Al-Harbi NO, Al-Harbi MM et al. Diosmin downregulates the expression of T cell receptors, pro-inflammatory cytokines and NF-KB activation against LPS-induced acute lung injury in mice. Pharmacol Res 2015; 102: 1-11

[5] Caly L, Druce JD, Catton MG et al. The FDA-approved Drug Ivermectin inhibits the replication of SARS-CoV-2 in vitro. Antiviral Research 2020; https://doi.org/10.1016/j. antiviral.2020.104787

[6] Hoffmann M, Kleine-Weber H, Schroeder S etal. SARS-CoV-2 cell entry depends on ACE2 and TMPRSS2 and is blocked by a clinically proven protease inhibitor. Cell 2020; 181: 271-280

[7] JM Lucas, Heinlein C, Kim T et al. The androgen-regulated protease TMPRSS2 activates a proteolytic cascade involving components of the tumor microenvironment and promotes prostate cancer metastasis. Cancer Discov 2014; 4: 1310-1325

Bibliography

DOI https://doi.org/10.1055/a-1170-4624

Published online: 19.5 .2020

Drug Res 2020; 70: 286

(c) Georg Thieme Verlag KG Stuttgart · New York ISSN 2194-9379

\section{Correspondence}

Dr. Maurizio Coppola

Department of Addiction, ASL CN2

Corso Coppino 46

12051 Alba

Italy

Tel.: +390173316210, Fax : +39017335067

mauriziocoppola1974@gmail.com 